\title{
Evaluation of Beer Fermentation with a Novel Yeast Williopsis saturnus
}

\author{
Shao-Quan Liu ${ }^{1,2 *}$ and Althea Ying Hui Quek ${ }^{1}$ \\ ${ }^{1}$ Food Science and Technology Programme, Department of Chemistry, National University of Singapore, \\ Science Drive 3, 117543 Singapore, Singapore \\ ${ }^{2}$ National University of Singapore (Suzhou) Research Institute, No. 377 Linquan Street, \\ Suzhou Industrial Park, 215123 Suzhou, PR China \\ Received: September 22, 2015 \\ Accepted: May 20, 2016
}

\begin{abstract}
Summary
The aim of this study is to evaluate the potential of a novel yeast Williopsis saturnus var. mrakii NCYC 500 to produce fruity beer. Fermentation performance of W. mrakii and beer volatile composition were compared against that fermented with Saccharomyces cerevisiae Safale US-05. ${ }^{\circ}$ Brix, sugar and $\mathrm{pH}$ differed significantly between the two types of beer. A total of 8 alcohols, 11 acids, 41 esters, 9 aldehydes, 8 ketones, 21 terpenes and terpenoids, 5 Maillard reaction products and 2 volatile phenolic compounds were detected. Yeast strain Safale US-05 was more capable of producing a wider range of ethyl and other esters, while yeast strain NCYC 500 produced significantly higher amounts of acetate esters. Strain NCYC 500 retained more terpenes and terpenoids, suggesting that the resultant beer could possess more of the aromatic hint of hops. This study showed that $W$. saturnus var. mrakii NCYC 500 could ferment wort to produce low-alcohol beer with higher levels of acetate esters, terpenes and terpenoids than yeast $S$. cerevisiae Safale US-05.
\end{abstract}

Key words: fermentation, beer, volatiles, esters, Williopsis sp., yeasts

\section{Introduction}

Beer is traditionally brewed from malted barley, water and hops using Saccharomyces cerevisiae, which produces flavour-active substances during wort fermentation, giving beer its flavour profile. These substances include esters, alcohols, aldehydes and ketones, volatile sulphur compounds and fatty acids, etc. Among the flavour volatiles, esters are one of the most important aroma compounds found in beer, which provide fruity aroma $(1,2)$.

Esters may be broadly categorised into two main groups, namely acetate esters and ethyl esters. Acetate esters are produced via alcoholysis from alcohols and acetyl coenzyme A (CoA) catalysed by alcohol acetyl transferases. Important acetate esters in beer include ethyl acetate, isoamyl acetate and 2-phenylethyl acetate (3). On the other hand, ethyl esters are secondary metabolites produced by both S. cerevisiae and non-Saccharomyces yeasts. These ethyl esters can be formed by esterification or alcoholysis. Esterification forms esters via condensation reactions between ethanol and carboxylic acids catalysed by fatty acid ethyl ester (FAEE) synthases. In contrast, alcoholysis is a transesterification reaction between fatty acyl-CoAs and ethanol catalysed by fatty acyl transferases (4). Important ethyl esters in beer include ethyl octanoate, ethyl hexanoate and ethyl decanoate (5).

At an industrial level, S. cerevisiae is the preferred yeast to perform beer fermentation because of its efficient conversion of sugars to ethanol, high ethanol tolerance and ability to carry out fermentation under acidic conditions as in wort (6). However, consistence in fermentation practices results in little flavour differentiation. To drive product differentiation, various microbreweries are dedi- 
cated to the production of specialty beer. Examples include fruit, honey and herb or spiced beer. To boost the fruity aroma in fruit beer, brewers have come up with different methods and styles of brewing by adding fruit (7). Fruits can also provide flavours, aromas and colours that cannot be obtained from grains.

The resultant fruit beer often lacks traditional beer flavours (7) so research has been directed at adding fruitiness to beer by enhancing the formation of flavour-active esters with the use of $S$. cerevisiae (1) or genetically modified yeasts $(4,5)$. However, given the controversial nature of genetic modification in food application, it would be of interest to impart fruitiness to beer using natural yeasts including non-Saccharomyces yeasts.

Non-Saccharomyces yeasts such as Brettanomyces sp. produce volatile phenols and play important positive roles in the spicy and smoky flavour profiles of specialty beer such as Lambic and Gueuze (8). Other non-Saccharomyces yeasts such as Candida and Pichia are well-known in sherry production for their role in oxidising ethanol to acetaldehyde (giving oxidised off-flavour) and have also been reported in mixed culture fermentation of acidic ales (8). Nevertheless, non-Saccharomyces yeasts can cause damage to beer and wine flavour, depending on the yeast and style of an alcoholic beverage.

One of the non-Saccharomyces yeasts that have received much research attention is from the genus Williopsis, which includes the saturn-shaped Williopsis saturnus (9). W. saturnus is known to possess high activities for synthesising fruity acetate esters, besides antifungal properties (10). Yilmaztekin et al. (11) studied the growth of $W$. saturnus var. saturnus on beet molasses and found that the yeast produced significant amounts of isoamyl acetate. Fermentation studies in sake using $W$. saturnus var. mrakii showed that it was a potent producer of acetate esters $(12,13)$.

Williopsis yeasts are not usually associated with beer fermentation, but have found potential applications in wine and sake fermentations (12-14). Furthermore, the ability of Williopsis yeasts to produce esters, especially acetate esters, while producing small amounts of ethanol in wine suggests a feasible way for production of fruity, low-alcohol beer (14). Therefore, the aim of this study is to evaluate the potential of $W$. saturnus var. mrakii NCYC 500 to produce fruity beer. Fermentation performance of W. mrakii NCYC 500 was compared against that of S. cerevisiae Safale US-05. Findings of this study could offer a novel biotechnological direction in brewing extra fruity, low-alcohol beer.

\section{Materials and Methods}

\section{Yeast strains and culture media}

Saccharomyces cerevisiae Safale US-05 (Fermentis Division of S. I. Lesaffre, Marcq-en-Baroeul, France) and Williopsis saturnus var. mrakii NCYC 500 (National Collection of Yeast Cultures, Norwich, UK) were used in this study. S. cerevisiae Safale US-05 was stored in its unopened, new package at $4{ }^{\circ} \mathrm{C}$. W. saturnus var. mrakii NCYC 500 was grown in malt-peptone-yeast extract-glucose broth $(\mathrm{pH}=5)$ consisting of (in \%, by mass per volume): glucose 2 , bacteriological peptone 0.25 , malt extract 0.25 and yeast extract 0.25 . Precultures were prepared by incubating the yeast in the same broth at $25^{\circ} \mathrm{C}$ for $48 \mathrm{~h}$. Strain NCYC 500 with 20 $\%$ glycerol was maintained at $-80{ }^{\circ} \mathrm{C}$ prior to use. Potato dextrose agar (PDA, $39 \mathrm{~g} / \mathrm{L}$; Oxoid, Basingstoke, Hampshire, UK) was used for enumeration.

\section{Wort preparation}

Wort was prepared by dissolving $1.1 \mathrm{~L}$ of Light Malt Extract (Coopers Brewery, Regency Park, Australia) in $6 \mathrm{~L}$ of water. A mass of $450 \mathrm{~g}$ of milled specialty malt (150 g of Carapils, $150 \mathrm{~g}$ of Crystal 10L and $150 \mathrm{~g}$ of Aromatic; Briess Malt \& Ingredients Co., Chilton, WI, USA) was steeped in $1.5 \mathrm{~L}$ of water at $70^{\circ} \mathrm{C}$ for $30 \mathrm{~min}$ for extraction of flavour compounds from the grains. The extract was then added to the dissolved malt extract to make up $8 \mathrm{~L}$ of solution, which was boiled for $60 \mathrm{~min}$. Once hot break had occurred, $113 \mathrm{~g}$ of glucose (brewing sugar) were mixed into the wort. Then, kettle hopping was carried out. At $60 \mathrm{~min}$ before the end of boiling, $14 \mathrm{~g}$ of Cascade hop pellets (iBrew, Schaumburg, IL, USA) were added to the wort. At $5 \mathrm{~min}$ before the end of boiling, $7 \mathrm{~g}$ of Perle hop pellets (iBrew) were added. After boiling, cold distilled water (Ice Mountain, Singapore) was added to the remaining wort to make up to $5 \mathrm{~L}$. The wort was then rapidly cooled down. Cooled wort was then bottled into 240-mL bottles and stored at $4{ }^{\circ} \mathrm{C}$. Before inoculation, the wort was centrifuged (29 703×g, 3-18K centrifuge; Sigma Laborzentrifugen $\mathrm{GmbH}$, Osterode am Harz, Germany) for $15 \mathrm{~min}$ and the supernatant was collected. Sterile filtration was carried out via peristaltic pumping of wort through a sterile $0.65-\mu \mathrm{m}$ filter and then a sterile $0.45-\mu \mathrm{m}$ filter.

\section{Fermentation conditions}

Triplicate fermentations were carried out in 500-mL sterile conical flasks (plugged with cotton wool, then wrapped in aluminium foil and parafilm) containing 400 $\mathrm{mL}$ of sterile wort at $21^{\circ} \mathrm{C}$. Inoculation conditions were as follows. For Safale US-05 strain, yeast cream was prepared following manufacturer's instructions: $0.92 \mathrm{~g}$ of dry yeast was reconstituted in 10 times its mass in wort $(8$ $\mathrm{mL}$ ). Yeast cream was then inoculated at $0.5 \%$, by volume (equivalent to approx. $10^{7} \mathrm{CFU} / \mathrm{mL}$ ). A preculture of NCYC 500 strain grown for $48 \mathrm{~h}$ in nutrient broth was inoculated at $1 \%$, by volume (equivalent to approx. $10^{5}$ $\mathrm{CFU} / \mathrm{mL}$ ). Fermentation was carried out statically for 14 days.

\section{Measurement of $\mathrm{pH}$ and ${ }^{\circ} \mathrm{Brix}$}

The total soluble solids ( ${ }^{\circ}$ Brix) and $\mathrm{pH}$ were measured using a refractometer (ATAGO Co., Ltd, Tokyo, Japan) and a pH meter (Metrohm, Zofingen, Switzerland), respectively.

\section{Analysis of sugars and organic acids}

Sugars were separated and quantified using a Shimadzu UFLC (Shimadzu, Kyoto, Japan) equipped with a Pinnacle II amino column ( $150 \mathrm{~mm} \times 4.6 \mathrm{~mm}$; Restek, Bellefonte, PA, USA). The mobile phase consisted of acetoni- 
trile and water (80:20) that was sonicated for $30 \mathrm{~min}$ before use. Elution was carried out at $40{ }^{\circ} \mathrm{C}$ with flow rate of $1 \mathrm{~mL} / \mathrm{min}$ (isocratic mode). An evaporative light-scattering detector (ELSD; Shimadzu, Kyoto, Japan) was used as the detector.

Organic acids were determined by a Shimadzu UFLC (same as above), using a Supelcogel C-610H column (Supelco, Bellefonte, PA, USA). A photodiode array detector at UV spectrum $(210 \mathrm{~nm})$ was used. The mobile phase contained $0.1 \%$ (by volume) sulphuric acid that was sonicated for $30 \mathrm{~min}$ before use. Elution was carried out at 40 ${ }^{\circ} \mathrm{C}$ with flow rate of $0.4 \mathrm{~mL} / \mathrm{min}$ (isocratic mode).

\section{Volatile analysis by HS-SPME-GC-MS/FID}

Volatiles were analysed using headspace (HS) solid-phase microextraction (SPME) and a Carboxen/PDMS fibre (85 $\mu \mathrm{m}$; Supelco, Sigma-Aldrich, Barcelona, Spain). This was coupled with gas chromatograph-mass spectrometer (GC-MS) and flame ionisation detector (FID) (Agilent Technologies, Santa Clara, CA, USA). Sample size was $5 \mathrm{~mL}$ (with $\mathrm{pH}$ adjusted to 2.5 using $1 \mathrm{M} \mathrm{HCl}$ ). Each sample was extracted by HS-SPME at $60{ }^{\circ} \mathrm{C}$ for 50 min using 250-rpm agitation. Thermal desorption of the fibre was carried out at the injection port of Agilent 7890A gas chromatograph (Agilent Technologies) coupled to Agilent $5975 \mathrm{C}$ triple-axis $\mathrm{MS}$ at $250{ }^{\circ} \mathrm{C}$ for $3 \mathrm{~min}$. Elution and separation of compounds were carried out with heli$\mathrm{um}$ as the carrier gas in a capillary column (Agilent DB-FFAP) with dimensions of $60 \mathrm{~m} \times 0.25 \mathrm{~mm}$ i.d. The column had a $0.25-\mu \mathrm{m}$ coating of polyethylene glycol modified with nitroterephthalic acid. Oven temperature was held at $50{ }^{\circ} \mathrm{C}$ for $5 \mathrm{~min}$ before a gradual increase of $5{ }^{\circ} \mathrm{C}$ per min until $230{ }^{\circ} \mathrm{C}$, which was held for $30 \mathrm{~min}$. Volatiles were identified by comparing their mass spectra with NIST08 Mass Spectral Library (15) and Wiley 275 Library (16). Identification of volatiles was verified using standard compound and/or linear retention indices (LRI).

\section{Statistical analysis}

Data collected from triplicate fermentations were subjected to statistical analysis. To compare values, analysis of variance (ANOVA) using Microsoft Office Excel (Microsoft, Redmond, WA, USA) was used. If the p-value was below 0.05 , differences were deemed statistically significant.

\section{Results and Discussion}

\section{Yeast growth, total soluble solids and $p H$ changes}

The two yeasts showed significant differences in terms of growth, total soluble solid content ( ${ }^{\circ}$ Brix) and pH changes (Fig. 1). S. cerevisiae Safale US-05 reached the maximum cell count on day 2 at $4.44 \cdot 10^{7} \mathrm{CFU} / \mathrm{mL}$. The maximum $W$. saturnus var. saturnus NCYC 500 cell count of $2.26 \cdot 10^{7} \mathrm{CFU} / \mathrm{mL}$ was reached on day 6 (Fig. 1a). The total soluble solid content reduced only slightly in the beer fermented with NCYC 500 strain relative to that of the beer fermented with Safale US-05 strain; the latter total soluble solid content decreased from 13.8 to $5.9 \%$ by day 6 (Fig. 1b). The $\mathrm{pH}$ decrease in the beer fermented
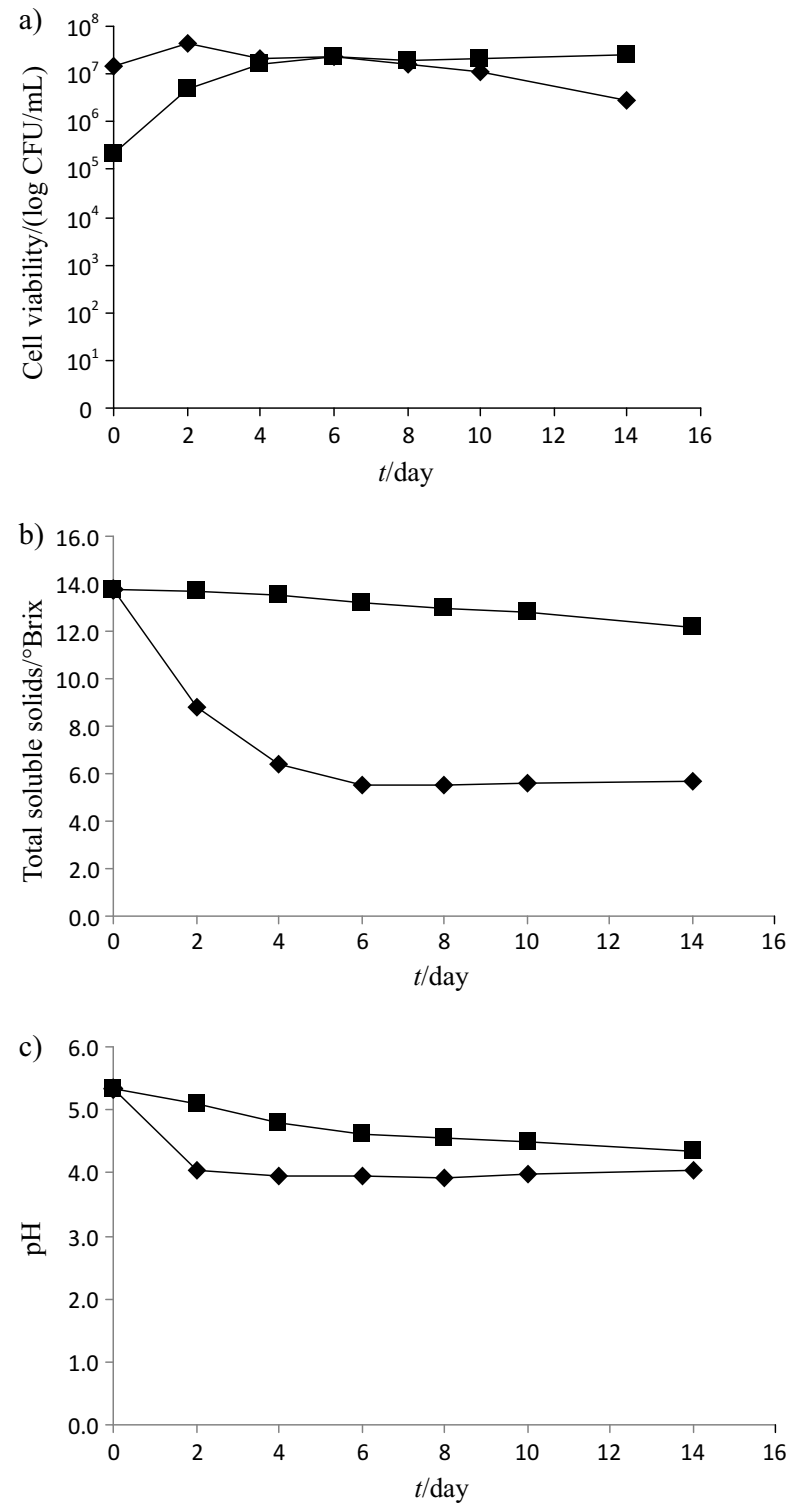

Fig. 1. Changes in: a) growth of yeasts, b) total soluble solids, and c) $\mathrm{pH}$ during ale beer fermentation by yeasts $S$. cerevisiae Safale US-05 (diamond) and W. saturnus var. mrakii NCYC 500 (square)

with NCYC 500 strain was more gradual than that of the beer fermented with Safale US-05 strain; the latter $\mathrm{pH}$ decreased markedly from day 0 to day 2 (Fig. 1c).

Other researchers have also observed the comparatively slower growth rates of Williopsis yeasts $(17,18)$. It has been shown that the intensity of fermentation (defined as the average number of days elapsing between inoculation of the glucose fermentation tube and the reduction of gas volume in the insert) with $W$. saturnus var. mrakii takes about 5 days (19). Thus, growth of NCYC 500 strain observed here corresponded to that reported for this yeast.

\section{Sugar utilisation}

Safale US-05 strain utilised glucose, fructose and sucrose rapidly by day 2 (Figs. $2 \mathrm{a}-\mathrm{c}$ ). This corresponded to 

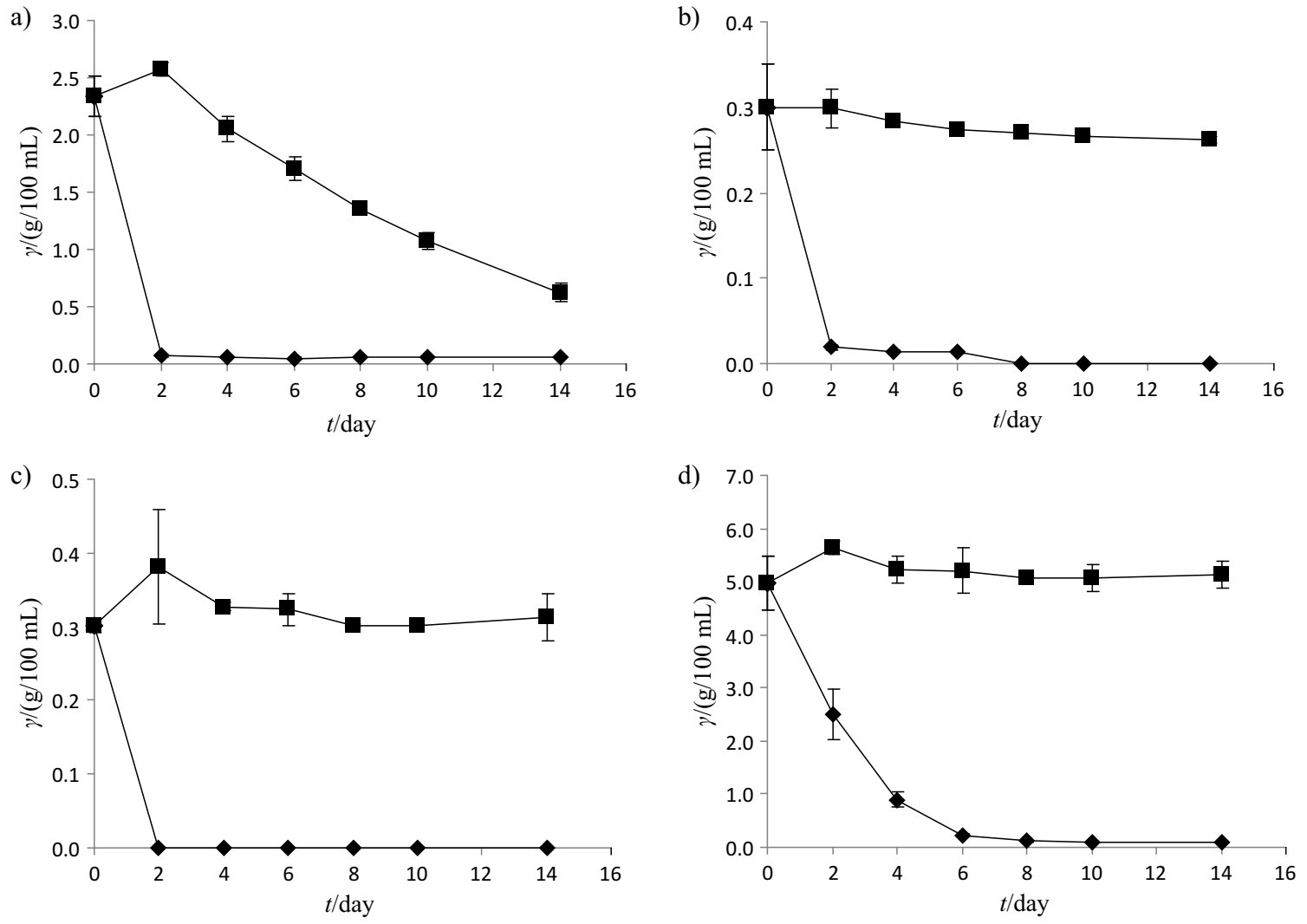

Fig. 2. Changes of: a) glucose, b) fructose, c) sucrose and d) maltose concentrations during ale beer fermentation by yeasts $S$. cerevisiae Safale US-05 (diamond) and W. saturnus var. mrakii NCYC 500 (square)

the large decreases in ${ }^{\circ} \mathrm{Brix}, \mathrm{pH}$ and maximal growth of Safale US-05 strain from day 0 to day 2, indicating the efficient fermentation of these sugars by the yeast. On the other hand, the utilisation of these sugars by NCYC 500 strain was much slower, with glucose being preferred but with few disaccharides being fermented. Maltose utilisation by Safale US-05 strain was the slowest amongst the four sugars (Fig. 2d), with only about half of the sugar being utilised by day 2 as opposed to the virtually complete utilisation of the other three sugars by the yeast during that time.

Utilisation of sugars by $S$. cerevisiae is well-studied with easily fermentable monosaccharides such as D-fructose and D-glucose, which are utilised first (20). Williopsis yeasts have also been reported to be glucophillic, consuming glucose faster than fructose (11). Sucrose was depleted by Safale US-05 strain by day 2 (Fig. 2c). It is not transported into the yeast cell but it is hydrolysed by invertase into fructose and glucose in the periplasmic space (21). Also, sucrose may be broken down via acid hydrolysis into the monosaccharides followed by intracellular utilisation. Unlike Safale US-05, the wort fermented by NCYC 500 strain had a high content of residual sucrose, likely due to its weak fermentative ability.

Utilisation of maltose by $S$. cerevisiae was slower than of fructose or glucose. This could be due to catabolite repression by glucose, which represses maltase gene expression (22). Fermentation of maltose with NCYC 500 strain was not significant (Fig. 2d). Non-utilisation of maltose is apparently characteristic of Williopsis saturnus yeasts (23). Maltose is a quantitatively dominant sugar in wort and its utilisation (or lack of) has a direct influence on the alcohol content in the final beer, as shown in this study.

\section{Organic acid metabolism}

Among the organic acids in beer fermented by Safale US-05 strain, those that showed significant increases in concentration were pyruvic, $\alpha$-ketoglutaric, lactic and acetic acids (Fig. 3). When using NCYC 500 strain, the pyruvic and acetic acid concentrations increased significantly. The concentration of the remaining acids stayed relatively constant (Fig. 3).

Pyruvic acid concentration increased significantly in the beer fermented with Safale US-05 strain (Fig. 3a). The beer fermented with NCYC 500 strain also showed an increase in pyruvic acid concentration, but it was not as significant. In both yeasts, the increase in pyruvic acid concentration was due to the Embden-Meyerhof pathway, which converts available sugars into pyruvic acid, yielding adenosine triphosphate (ATP). Fermentation rate by Safale US-05 was much faster than by NCYC 500 strain, which might explain why pyruvic acid concentration in the Safale US-05-fermented beer increased significantly by day 2, while there was a much more gradual increase in pyruvic acid concentration in the beer fermented with NCYC 500. In addition, the higher concentration of pyruvic acid in the beer fermented with Safale US-05 could also be because $S$. cerevisiae carries out anaerobic fermentation 


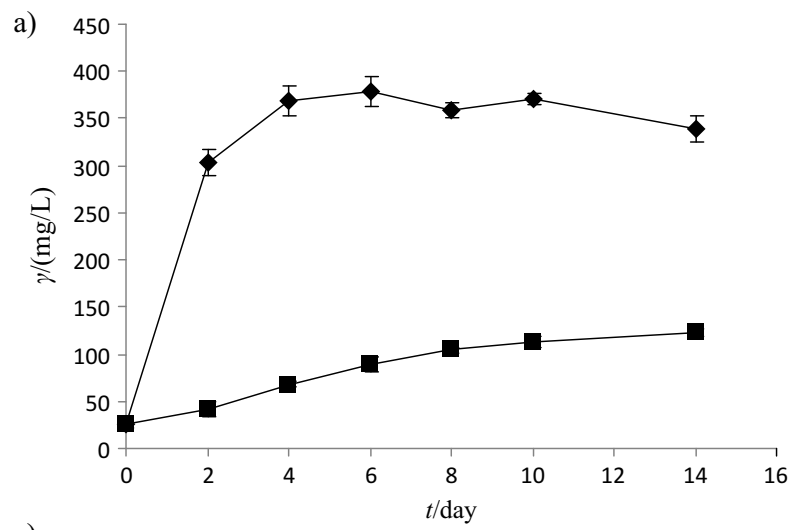

c)

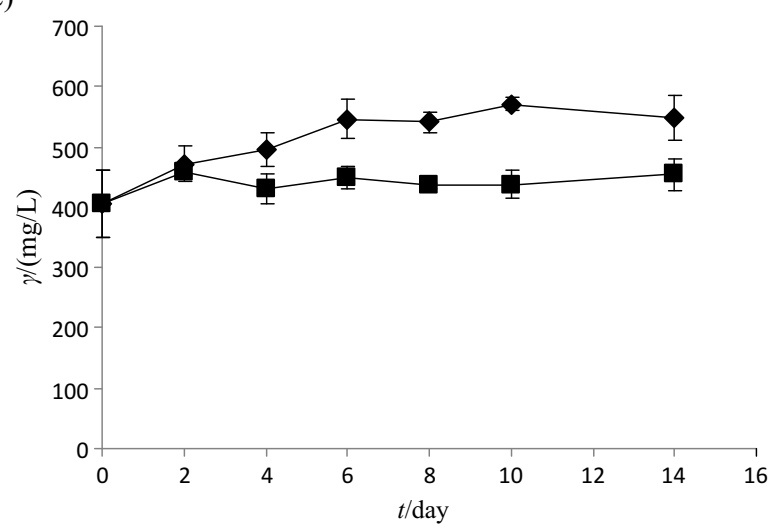

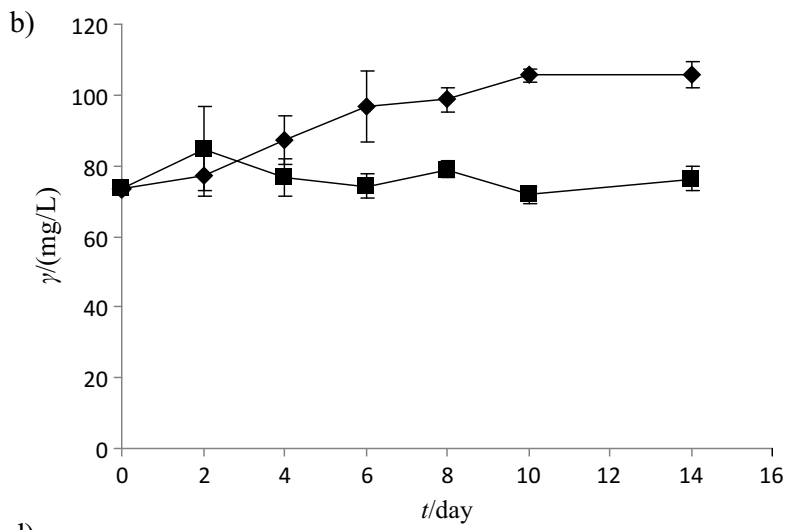

d)

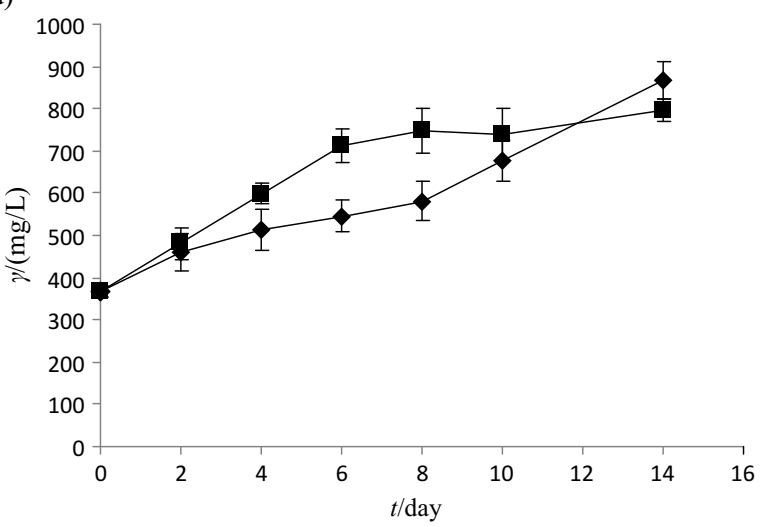

Fig. 3. Changes of major organic acid: a) pyruvic, b) $\alpha$-ketoglutaric, c) lactic and d) acetic acid concentrations during ale beer fermentation by S. cerevisiae Safale US-05 (diamond) and W. saturnus var. mrakii NCYC 500 (square)

while $W$. saturnus var. mrakii is more oxidative. Thus, oxidative respiration would result in a lower concentration of pyruvic acid being excreted into the medium by NCYC 500 strain.

The initial concentration of $\alpha$-ketoglutaric acid was low (Fig. 3b). In the beer fermented with Safale US-05 strain, $\alpha$-ketoglutaric acid concentration increased. In the beer fermented with NCYC 500 strain, $\alpha$-ketoglutaric acid concentration showed minor fluctuations. $\alpha$-Ketoglutaric acid is synthesised from the Krebs cycle or from amino acid catabolism and is a minor organic acid found in beer (24). Thus, it was expected that in the beer fermented with Safale US-05 the increase of the concentration of this acid was greater because amino acid catabolism was more extensive in S. cerevisiae than in NCYC 500 strain (data not shown).

Lactic acid concentration increased slightly throughout fermentation in the beer fermented with Safale US-05 (Fig. 3c). In the beer fermented with NCYC 500 strain, there were minor fluctuations in lactic acid concentration. Strain NCYC 500, being more oxidative, would likely carry out little, or none at all, anaerobic conversion of pyruvic to lactic acid, which might explain the insignificant concentration of lactic acid in the beer fermented with NCYC 500.

Acetic acid concentration in the beer fermented with both types of yeast increased, with the production rate being higher by NCYC-500 than by Safale US-05 (Fig. 3d). However, the final concentration of acetic acid was a little higher in the beer fermented with Safale US-05 $(870 \mathrm{mg} / \mathrm{L})$ than in the beer fermented with NCYC $500(800 \mathrm{mg} / \mathrm{L})$. Acetic acid can be formed in $S$. cerevisiae by the oxidation of acetaldehyde, an intermediate in the alcoholic fermentation pathway, and co-reduction of $\mathrm{NAD}^{+}$catalysed by aldehyde dehydrogenase. The oxidation of acetaldehyde to acetic acid acts as a redox sink for anabolic and physiological stress reactions. Pyruvic acid can also be reabsorbed by the yeast and metabolised to acetate (25). Excess acetic acid is secreted into the fermentation medium (26). Acetic acid is not desired in alcoholic beverages as it can impart a sour vinegary off-odour if its concentration is too high. Since the flavour threshold of acetic acid in beer is $130 \mathrm{mg} / \mathrm{L}$, the detected concentrations of acetic acid in the two beers could impart a vinegary odour, although this was not discernible organoleptically in informal sensory evaluation by sniffing.

\section{Changes in volatile composition}

A total of 8 alcohols, 11 acids, 41 esters, 9 aldehydes, 8 ketones, 21 terpenes and terpenoids, 5 Maillard reaction products (furans, pyrans and pyrroles) and 2 volatile phenolic compounds were detected in the beer samples. Selected major volatiles in wort and beer are presented in Table 1.

The biggest group of volatiles detected in the beer samples were esters (Table 1). Fermentation altered the volatile composition of wort drastically, with a large variety of esters reduced or produced via the yeast metabolism. Ethyl and acetate esters were the two most significant types of esters detected. 
Table 1. Selected major volatile compounds (GC-FID peak area $10^{6}$ ) and their relative peak areas (RPA) identified in wort and beer fermented with S. cerevisiae Safale US-05 or W. saturnus var. mrakii NCYC 500 after 14 days of fermentation

\begin{tabular}{|c|c|c|c|c|c|c|c|c|}
\hline \multirow{2}{*}{ Compound } & \multirow{2}{*}{ RI } & \multicolumn{2}{|c|}{ Wort } & \multicolumn{2}{|c|}{$\begin{array}{c}\text { Beer fermented } \\
\text { with Safale US-05 }\end{array}$} & \multicolumn{2}{|c|}{$\begin{array}{l}\text { Beer fermented } \\
\text { with NCYC } 500\end{array}$} & \multirow{2}{*}{ Organoleptic properties } \\
\hline & & Peak area & RPA/\% & Peak area & $\mathrm{RPA} / \%$ & Peak area & $\mathrm{RPA} / \%$ & \\
\hline \multicolumn{9}{|l|}{ Acids } \\
\hline Acetic & 1464 & $(2.2 \pm 0.3)^{\mathrm{a}}$ & 1.2 & $(4.8 \pm 0.2)^{\mathrm{b}}$ & 0.1 & $(7.4 \pm 0.4)^{\mathrm{c}}$ & 0.7 & Sour, vinegar \\
\hline Isobutanoic & 1576 & $(0.6 \pm 0.1)^{\mathrm{a}}$ & 0.3 & $(1.2 \pm 0.3)^{\mathrm{a}}$ & 0.03 & $(0.5 \pm 0.1)^{\mathrm{a}}$ & 0.04 & Acidic, unpleasant \\
\hline Hexanoic & 1855 & $(0.6 \pm 0.0)^{\mathrm{a}}$ & 0.3 & $(4.5 \pm 0.7)^{\mathrm{b}}$ & 0.1 & $(1.6 \pm 0.1)^{\mathrm{a}}$ & 0.2 & Sour, fatty, cheesy \\
\hline Octanoic & 2068 & $(1.8 \pm 0.4)^{\mathrm{a}}$ & 1.0 & $(28.2 \pm 6.5)^{\mathrm{b}}$ & 0.7 & $(3.6 \pm 0.1)^{\mathrm{a}}$ & 0.3 & Fatty, rancid, cheesy \\
\hline Decanoic & 2282 & $(0.6 \pm 0.1)^{\mathrm{a}}$ & 0.3 & $(9.2 \pm 2.9)^{\mathrm{b}}$ & 0.2 & $(1.2 \pm 0.1)^{\mathrm{a}}$ & 0.1 & Rancid, sour, fatty \\
\hline Benzoic & 2463 & $(0.2 \pm 0.1)^{\mathrm{a}}$ & 0.1 & $(0.8 \pm 0.1)^{\mathrm{b}}$ & 0.02 & $(0.2 \pm 0.0)^{\mathrm{a}}$ & 0.02 & Faint, balsam \\
\hline \multicolumn{9}{|l|}{ Alcohols } \\
\hline Ethanol & 946 & $(8.1 \pm 0.3)^{\mathrm{a}}$ & 4.3 & $(3052 \pm 133)^{b}$ & 77.4 & $(604 \pm 33)^{c}$ & 57.0 & Alcoholic, ethereal \\
\hline Isobutanol & 1084 & n.d. & 0.0 & $(8.9 \pm 0.3)^{\mathrm{a}}$ & 0.2 & $(5.2 \pm 0.2)^{\mathrm{b}}$ & 0.5 & Ethereal, winey \\
\hline Active amyl alcohol & 1211 & $(0.8 \pm 0.) 2^{\mathrm{a}}$ & 0.4 & n.d. & 0.0 & $(7.1 \pm 0.5)^{\mathrm{b}}$ & 0.7 & Pungent, fermented \\
\hline Isoamyl alcohol & 1214 & $(4.3 \pm 0.2)^{\mathrm{a}}$ & 2.3 & $(25.4 \pm 5.4)^{\mathrm{b}}$ & 0.6 & $(44.7 \pm 4.1)^{c}$ & 4.2 & Fusel, alcoholic, fruity \\
\hline Hexanol & 1359 & $(0.67 \pm 0.01)^{\mathrm{a}}$ & 0.4 & $(0.21 \pm 0.02)^{\mathrm{b}}$ & 0.01 & $(0.8 \pm 0.1)^{\mathrm{a}}$ & 0.07 & Pungent, fusel oil, fruity \\
\hline 2-Phenethyl alcohol & 2027 & n.d. & 0.0 & $(56.3 \pm 3.8)^{\mathrm{a}}$ & 1.4 & $(1.1 \pm 0.0)^{\mathrm{b}}$ & 0.1 & Floral, rose, honey \\
\hline \multicolumn{9}{|l|}{ Aldehydes } \\
\hline Acetaldehyde & 761 & n.d. & 0.0 & $9.7 \pm 0.4$ & 0.3 & n.d. & 0.0 & Pungent, ethereal, fruity \\
\hline 3-Methylbutanal & 929 & $9.0 \pm 0.6$ & 4.8 & n.d. & 0.0 & n.d. & 0.0 & Ethereal, chocolate, peach \\
\hline Hexanal & 1067 & $0.91 \pm 0.07$ & 0.5 & n.d. & 0.0 & n.d. & 0.0 & Fresh, green, fatty \\
\hline Nonanal & 1390 & n.d. & 0.0 & $(1.7 \pm 0.2)^{\mathrm{a}}$ & 0.04 & $(0.81 \pm 0.04)^{\mathrm{b}}$ & 0.1 & Fatty, orange peel \\
\hline Benzaldehyde & 1543 & $(2.7 \pm 0.2)^{\mathrm{a}}$ & 1.5 & $(4.3 \pm 0.2)^{\mathrm{b}}$ & 0.1 & $(4.0 \pm 0.2)^{\mathrm{b}}$ & 0.4 & Almond, cherry \\
\hline (E)-2-Decenal & 1655 & n.d. & 0.0 & $(0.36 \pm 0.02)^{\mathrm{a}}$ & 0.01 & $(0.29 \pm 0.03)^{\mathrm{b}}$ & 0.03 & Waxy, coriander \\
\hline 2-Phenylacetaldehyde & 1665 & $1.8 \pm 0.0$ & 1.0 & n.d. & 0.0 & n.d. & 0.0 & Green, floral, honey \\
\hline$o$-Tolualdehyde & 1673 & n.d. & 0.0 & $(1.7 \pm 0.2)^{\mathrm{a}}$ & 0.04 & $(4.9 \pm 0.1)^{\mathrm{b}}$ & 0.5 & Cherry \\
\hline$m$-Tolualdehyde & 1673 & $6.0 \pm 0.2$ & 3.2 & n.d. & 0.0 & n.d. & 0.0 & Sweet, cherry \\
\hline \multicolumn{9}{|l|}{ Esters } \\
\hline Methyl heptanoate & 1277 & $0.24 \pm 0.02$ & 0.1 & n.d. & 0.0 & n.d. & 0.0 & Sweet, fruity \\
\hline Methyl 4-methyl-2-hexenoate & 1329 & n.d. & 0.0 & $(0.55 \pm 0.06)^{\mathrm{a}}$ & 0.01 & $(2.7 \pm 0.1)^{\mathrm{b}}$ & 0.3 & Sweet, fruity \\
\hline Ethyl propionate & 958 & $3.7 \pm 0.2$ & 2.0 & n.d. & 0.0 & n.d. & 0.0 & Sweet, grape, winey \\
\hline Ethyl butanoate & 1034 & n.d. & 0.0 & $(5.6 \pm 0.4)^{\mathrm{a}}$ & 0.14 & $(3.4 \pm 0.1)^{\mathrm{b}}$ & 0.3 & Fruity, pineapple \\
\hline Ethyl hexanoate & 1216 & n.d. & 0.0 & $42.4 \pm 7.5$ & 1.1 & n.d. & 0.0 & Sweet, fruity, pineapple \\
\hline Ethyl octanoate & 1435 & $(1.6 \pm 0.3)^{\mathrm{a}}$ & 0.9 & $(383 \pm 6.0)^{b}$ & 9.7 & $(3.05 \pm 0.02)^{\mathrm{a}}$ & 0.3 & Fruity, wine, sweet \\
\hline Ethyl decanoate & 1643 & $(2.2 \pm 0.8)^{\mathrm{a}}$ & 1.2 & $(182 \pm 41.5)^{\mathrm{b}}$ & 4.6 & $(5.46 \pm 0.05)^{\mathrm{a}}$ & 0.5 & Sweet, fruity, apple, grape \\
\hline Ethyl 9-decenoate & 1694 & $(0.5 \pm 0.1)^{\mathrm{a}}$ & 0.2 & $(45.1 \pm 8.9)^{\mathrm{b}}$ & 1.1 & $(0.28 \pm 0.03)^{\mathrm{a}}$ & 0.03 & Fruity, fatty \\
\hline Ethyl laurate & 1850 & $(0.4 \pm 0.2)^{\mathrm{a}}$ & 0.2 & $(16.7 \pm 5.6)^{\mathrm{b}}$ & 0.4 & $(3.0 \pm 0.2)^{\mathrm{a}}$ & 0.3 & Sweet, waxy, soapy \\
\hline Isobutyl isobutanoate & 1070 & $(7.3 \pm 0.1)^{\mathrm{a}}$ & 3.9 & n.d. & 0.0 & $(0.44 \pm 0.03)^{\mathrm{b}}$ & 0.04 & Pineapple, tropical \\
\hline Isobutyl 2-methylbutanoate & 1148 & $0.29 \pm 0.04$ & 0.2 & n.d. & 0.0 & n.d. & 0.0 & Sweet, fruity \\
\hline Isoamyl propionate & 1165 & $(4.6 \pm 0.1)^{\mathrm{a}}$ & 2.4 & n.d. & 0.0 & $(3.3 \pm 0.2)^{\mathrm{b}}$ & 0.3 & Sweet, fruity, pineapple \\
\hline Amyl isobutanoate & 1170 & $1.5 \pm 0.1$ & 0.8 & n.d. & 0.0 & n.d. & 0.0 & Fruity, apricot, pineapple \\
\hline 2-Methylbutyl isobutanoate & 1172 & $8.0 \pm 0.1$ & 4.3 & n.d. & 0.0 & n.d. & 0.0 & Fruity, ethereal \\
\hline Isoamyl octanoate & 1455 & n.d. & 0.0 & $0.49 \pm 0.02$ & 0.01 & n.d. & 0.0 & Sweet, pineapple, coconut \\
\hline Propyl octanoate & 1516 & n.d. & 0.0 & $0.66 \pm 0.06$ & 0.02 & n.d. & 0.0 & Coconut, cacao, gin \\
\hline 2-Methylbutyl octanoate & 1660 & n.d. & 0.0 & $2.6 \pm 0.5$ & 0.07 & n.d. & 0.0 & Apple, fruity \\
\hline Propyl decanoate & 1725 & n.d. & 0.0 & $0.22 \pm 0.05$ & 0.01 & n.d. & 0.0 & Fruity, fatty, green \\
\hline Isobutyl decanoate & 1760 & n.d. & 0.0 & $0.30 \pm 0.07$ & 0.01 & n.d. & 0.0 & Brandy, apricot, cognac \\
\hline Isoamyl decanoate & 1867 & n.d. & 0.0 & $1.3 \pm 0.2$ & 0.03 & n.d. & 0.0 & Fruity, sweet \\
\hline
\end{tabular}


Table 1. - continued

\begin{tabular}{|c|c|c|c|c|c|c|c|c|}
\hline \multirow{2}{*}{ Compound } & \multirow{2}{*}{ RI } & \multicolumn{2}{|c|}{ Wort } & \multicolumn{2}{|c|}{$\begin{array}{l}\text { Beer fermented } \\
\text { with Safale US-05 }\end{array}$} & \multicolumn{2}{|c|}{$\begin{array}{l}\text { Beer fermented } \\
\text { with NCYC } 500\end{array}$} & \multirow{2}{*}{ Organoleptic properties } \\
\hline & & Peak area & $\mathrm{RPA} / \%$ & Peak area & $\mathrm{RPA} / \%$ & Peak area & $\mathrm{RPA} / \%$ & \\
\hline Ethyl acetate & 905 & $(19.7 \pm 0.7)^{\mathrm{a}}$ & 10.42 & $(14.7 \pm 0.9)^{\mathrm{a}}$ & 0.4 & $(155 \pm 9)^{\mathrm{b}}$ & 14.6 & $\begin{array}{l}\text { Ethereal, fruity, sweet, } \\
\text { weedy, green }\end{array}$ \\
\hline Isobutyl acetate & 1015 & n.d. & 0.0 & n.d. & 0.0 & $3.8 \pm 0.3$ & 0.4 & Sweet, fruity, ethereal \\
\hline Isoamyl acetate & 1095 & $(45.8 \pm 1.0)^{\mathrm{a}}$ & 24.3 & $(7.8 \pm 0.6)^{\mathrm{b}}$ & 0.2 & $(148 \pm 14.5)^{\mathrm{c}}$ & 14.0 & Sweet, fruity, banana \\
\hline 2-Phenylethyl acetate & 1832 & $(0.8 \pm 0.0)^{\mathrm{a}}$ & 0.4 & $(2.9 \pm 0.5)^{\mathrm{a}}$ & 0.07 & $(12.9 \pm 1.8)^{\mathrm{b}}$ & 1.2 & Floral, rose, sweet \\
\hline \multicolumn{9}{|l|}{ Ketones } \\
\hline 4-Methyl-2-pentanone & 1012 & $3.2 \pm 0.1$ & 1.7 & n.d. & 0.00 & n.d. & 0.00 & Sharp, green, fruity \\
\hline 2-Octanone & 1280 & $(0.37 \pm 0.03)^{\mathrm{a}}$ & 0.2 & n.d. & 0.00 & $(0.16 \pm 0.01)^{\mathrm{b}}$ & 0.01 & Earthy, woody \\
\hline 6-Methyl-5-hepten-2-one & 1338 & $(1.05 \pm 0.04)^{\mathrm{a}}$ & 0.6 & $(0.34 \pm 0.05)^{\mathrm{b}}$ & 0.01 & $(0.220 \pm 0.004)^{\mathrm{b}}$ & 0.02 & Mushroom, blackcurrant \\
\hline 2-Nonanone & 1389 & $(2.1 \pm 0.3)^{\mathrm{a}}$ & 1.1 & $(0.41 \pm 0.08)^{\mathrm{b}}$ & 0.01 & n.d. & 0.00 & Fruity, sweet, waxy \\
\hline \multicolumn{9}{|l|}{ Maillard products } \\
\hline 2-Acetylpyrrole & 1996 & $(0.11 \pm 0.01)^{\mathrm{a}}$ & 0.06 & $(0.17 \pm 0.02)^{\mathrm{b}}$ & 0.00 & $(0.150 \pm 0.004)^{\mathrm{b}}$ & 0.01 & Musty, nutty \\
\hline Furfural & 1481 & $(9.6 \pm 0.3)^{a}$ & 5.1 & $(0.53 \pm 0.05)^{\mathrm{b}}$ & 0.01 & $(1.69 \pm 0.04)^{c}$ & 0.2 & Sweet, caramellic \\
\hline 2-Acetylfuran & 1523 & $(0.50 \pm 0.07)^{\mathrm{a}}$ & 0.3 & $(0.23 \pm 0.02)^{\mathrm{b}}$ & 0.01 & $(0.67 \pm 0.04)^{c}$ & 0.06 & Sweet, caramel, coffee \\
\hline \multicolumn{9}{|l|}{ Phenolic compounds } \\
\hline 4-Vinyl-2-methoxy-phenol & 2225 & $(0.080 \pm 0.004)^{\mathrm{a}}$ & 0.04 & $(0.15 \pm 0.02)^{\mathrm{b}}$ & 0.00 & $(0.100 \pm 0.002)^{\mathrm{a}}$ & 0.01 & Spicy, clove, smoky \\
\hline 2,4-di-tert-Butylphenol & 2322 & $(0.90 \pm 0.02)^{\mathrm{a}}$ & 0.48 & $(0.22 \pm 0.02)^{\mathrm{b}}$ & 0.01 & $(0.49 \pm 0.01)^{c}$ & 0.05 & Phenolic \\
\hline Dimethyl disulfide & 1061 & $1.17 \pm 0.04$ & 0.62 & n.d. & 0.00 & n.d. & 0.00 & Sulfurous, cabbage \\
\hline \multicolumn{9}{|l|}{ Terpenes and terpenoids } \\
\hline$\alpha$-Phellandrene & 1126 & $1.6 \pm 0.2$ & 0.8 & n.d. & 0.00 & n.d. & 0.00 & Citrus, green \\
\hline Sabinene & 1128 & $(0.6 \pm 0.1)^{\mathrm{a}}$ & 0.3 & $(0.26 \pm 0.05)^{\mathrm{b}}$ & 0.01 & $(1.79 \pm 0.01)^{c}$ & 0.17 & Woody, citrus \\
\hline$\alpha$-Terpinene & 1142 & $(0.40 \pm 0.05)^{\mathrm{a}}$ & 0.2 & $(0.15 \pm 0.02)^{\mathrm{b}}$ & 0.00 & $(0.38 \pm 0.01)^{\mathrm{a}}$ & 0.04 & Citrusy, woody \\
\hline Limonene & 1161 & n.d. & 0.0 & $(0.39 \pm 0.07)^{\mathrm{a}}$ & 0.01 & $(0.45 \pm 0.15)^{\mathrm{a}}$ & 0.04 & Citrus, fresh, sweet \\
\hline cis-Ocimene & 1208 & $1.2 \pm 0.4$ & 0.6 & n.d. & 0.00 & n.d. & 0.00 & Citric, floral, sweet \\
\hline trans- $\beta$-Ocimene & 1229 & $(0.93 \pm 0.05)^{\mathrm{a}}$ & 0.5 & $(0.33 \pm 0.11)^{\mathrm{b}}$ & 0.01 & $(0.66 \pm 0.05)^{\mathrm{c}}$ & 0.06 & Sweet, herbal \\
\hline$p$-Cymene & 1253 & n.d. & 0.0 & $(0.20 \pm 0.07)^{\mathrm{a}}$ & 0.00 & $(0.68 \pm 0.06)^{b}$ & 0.06 & Fresh, citrus, woody \\
\hline$\alpha$-Terpinolene & 1262 & $(0.37 \pm 0.04)^{\mathrm{a}}$ & 0.2 & n.d. & 0.00 & $(0.24 \pm 0.02)^{\mathrm{b}}$ & 0.02 & Fresh, woody, citrus \\
\hline Linalool oxide & 1448 & $(0.27 \pm 0.05)^{\mathrm{a}}$ & 0.14 & n.d. & 0.00 & $(0.130 \pm 0.003)^{\mathrm{b}}$ & 0.01 & Floral, herbal, green \\
\hline Linalool & 1550 & $(14.8 \pm 0.3)^{\mathrm{a}}$ & 7.9 & $(0.99 \pm 0.11)^{\mathrm{b}}$ & 0.03 & $(10.9 \pm 0.2)^{c}$ & 1.03 & Citrus, floral, waxy \\
\hline$\beta$-Caryophyllene & 1597 & $0.33 \pm 0.05$ & 0.2 & n.d. & 0.00 & n.d. & 0.00 & Sweet, woody, clove \\
\hline 4-Terpineol & 1611 & $(0.65 \pm 0.03)^{\mathrm{a}}$ & 0.4 & n.d. & 0.00 & $(0.17 \pm 0.01)^{\mathrm{b}}$ & 0.02 & Woody, mentholic, citrus \\
\hline Citronellyl acetate & 1664 & n.d. & 0.0 & $(0.65 \pm 0.01)^{\mathrm{a}}$ & 0.02 & $(0.43 \pm 0.02)^{\mathrm{b}}$ & 0.04 & Floral, green, rose, citrus \\
\hline$\alpha$-Terpineol & 1710 & $(1.46 \pm 0.08)^{\mathrm{a}}$ & 0.8 & $(0.44 \pm 0.05)^{\mathrm{b}}$ & 0.01 & $(0.96 \pm 0.05)^{\mathrm{c}}$ & 0.09 & Pine, citrus, floral \\
\hline$\alpha$-Selinene & 1730 & $0.12 \pm 0.04$ & 0.07 & n.d. & 0.00 & n.d. & 0.00 & Amber \\
\hline Geranyl acetate & 1762 & n.d. & 0.0 & n.d. & 0.00 & $0.44 \pm 0.05$ & 0.04 & Floral, rose, green \\
\hline trans- $\beta$-Damascenone & 1835 & $(1.83 \pm 0.09)^{\mathrm{a}}$ & 1.0 & $(0.56 \pm 0.03)^{\mathrm{b}}$ & 0.01 & $(1.38 \pm 0.08)^{c}$ & 0.13 & Apple, rose, honey \\
\hline
\end{tabular}

Statistical analysis at $95 \%$ confidence level with same letters in superscript indicates no significant difference;

$\mathrm{RI}=$ experimentally determined retention index on the DB-FFAP column, relative to C5-C40 hydrocarbons; n.d.=not detected

In the wort, there were only a few ethyl esters detected. However, Safale US-05 strain produced a wider variety of ethyl esters in higher concentrations relative to NCYC 500 strain after fermentation. Major ethyl esters were ethyl octanoate, decanoate, 9-decenoate and laurate. Fatty acid ethyl esters are secondary metabolites produced by $S$. cerevisiae (4). Esterification of the medium-chain fatty acids that are thought to be toxic to yeast can be considered as a means to allow the diffusion of these toxic compounds out of the cell (4). In terms of flavour, ethyl octanoate with a very low detection threshold is expected to contribute significantly to the desired fruity aspect of beer (3).

Acetate esters were the next major type of esters formed by strains Safale US-05 and NCYC 500. The major acetate esters were ethyl, isoamyl and 2-phenylethyl acetate. The concentrations of ethyl and isoamyl acetate de- 
tected in the beer fermented with NCYC 500 strain were significantly higher than those detected in the beer fermented with Safale US-05. Williopsis yeasts are able to produce high concentrations of acetate esters, in particular isoamyl, ethyl and 2-phenylethyl acetate $(11,12)$. The concentration of isoamyl acetate in the beer fermented with Safale US-05 was approx. 20 times lower than in that fermented with NCYC 500. 2-Phenylethyl acetate production by Safale US- 05 was 5 times lower than that by NCYC 500 strain. Thus, usage of Williopsis to ferment wort would result in a beer with a more distinct fruity, banana-like and floral aroma imparted by higher concentrations of acetate esters (11). However, the higher production of ethyl acetate by NCYC 500 strain could lead to a glue-like off-flavour in beer (27).

Methyl esters were produced at low concentrations in both beer samples fermented with Safale US-05 and NCYC 500. These methyl esters were likely products of the esterification of methanol and organic acids. The presence of methanol in fruit and grain-based beverages has been attributed to the demethylation of methylated pectins, possibly due to acid hydrolysis (28) or enzyme action.

The major alcohol produced by both yeasts was ethanol, with Safale US-05 strain producing about 5 times more ethanol than NCYC 500 strain (Table 1). Based on ${ }^{\circ}$ Brix approximation, the ethanol volume fraction in the beer fermented with Safale US-05 was $6.9 \%$, while that of the beer fermented with NCYC 500 strain was $1.7 \%$. This result was expected, as NCYC 500 strain has been reported to carry out fermentation with low ethanol production $(11,29)$. Williopsis yeasts seem to predominantly utilize sugars via oxidative respiration, producing carbon dioxide and water, but only little ethanol (29). Other major alcohols detected in both beer samples included isobutanol, active amyl, isoamyl and 2-phenethyl alcohol, which are mainly produced via branched-chain and aromatic amino acid metabolism (30).

Short- to medium-chain fatty acids such as hexanoic, octanoic and decanoic acids were detected in the highest concentrations in both beer samples (Table 1). The fatty acids may be products of yeast metabolism such as $\beta$-oxidation of higher fatty acids (31). These fatty acids are important for beer flavour because they themselves are odour-active and can lead to rancid off-flavour at high concentrations. Moreover, they are also one of the starting materials for the synthesis of volatile esters (32). In general, fatty acid production by Safale US-05 was higher than by NCYC 500 strain.

Terpenes and terpenoids in wort are mainly derived from added hops. Terpene and terpenoid concentrations generally decreased from day 0 to day 14 in the beer fermented with either Safale US-05 or NCYC 500, with some terpenes becoming undetectable (Table 1). The exception was $p$-cymene, which was not detected in the wort, but was detected in both beer samples fermented with US-05 or NCYC 500 strain.

The most significant terpenoid detected in the wort and the fermented beer samples was linalool (Table 1). Linalool is one of the key odourants that impart a floral, fruity and citrus note to the Cascade and Perle hops
$(33,34)$ used in this study. More importantly, linalool is also one of the hop-derived odourants in hopped beer, indicating that the presence of this terpenoid in beer comes at least partly from wort $(35,36)$. However, the concentration of linalool found in the beer can be affected by yeast metabolism (37). S. cerevisiae is able to convert the odourless linalyl glycoside to linalool via the action of $\beta$-glucosidases (38). Yeast has been shown to be able to convert geraniol to linalool $(37,39)$, although geraniol was not detected in the wort.

Linalool content could also decrease due to yeast metabolism, as indicated by our results (Table 1). It has been shown that linalool can be converted into other terpenoids such as $\alpha$-terpineol $(37,39)$, which was detected in the final beer samples albeit at levels lower than those in the wort. Linalool could also have been transformed into linalool oxide detected in the beer fermented with NCYC 500 strain. Hence, it must be pointed out that the final concentration of linalool is a net balance of production and utilisation.

Two terpenoid esters were detected in the fermented beer, but not in the wort. Specifically, citronellyl acetate was detected in both beer samples fermented with Safale US-05 and NCYC 500, but geranyl acetate was detected only in the beer fermented with NCYC 500 strain (Table 1). Citronellol was not detected in the wort, but it is possible that citronellyl acetate could have been formed via reduction of geranyl acetate (37). In addition, although geraniol was also not detected in all the samples, geranyl acetate was still formed. It has been shown that the geraniol to linalool biotransformation reaction is reversible; thus, some linalool present in the wort could have been converted into geraniol via yeast metabolism (37). Strain NCYC 500, with its higher acetate ester-synthesising activity than that of Safale US-05, could then form geranyl acetate, whereas the latter could not.

Acetaldehyde was the major aldehyde detected in the beer fermented with Safale US-05 strain, but it was not detected in the beer fermented with NCYC 500 strain (Table 1). In S. cerevisiae, anaerobic catabolism of sugars to produce ethanol involves production of acetaldehyde as an intermediate. Although most of the acetaldehyde is metabolised, some molecules would still diffuse out of the cell $(40)$. Strain NCYC 500 has a low anaerobic fermentative ability; hence, the alcoholic fermentative pathway would not be carried out as extensively as by Safale US-05, resulting in only trace amounts of acetaldehyde formed.

Benzaldehyde was also another major aldehyde detected in the beer fermented by either Safale US-05 or NCYC 500 (Table 1). Benzaldehyde may originate from the oxidative degradation of phenylpyruvic acid during phenylalanine metabolism of yeast (41). 3-Methylbutanal, hexanal, 2-phenylacetaldehyde and $m$-tolualdehyde (3-methylbenzaldehyde) were detected in the wort but not in the beer, probably due to yeast-mediated reduction of aldehydes to alcohols. Nonanal, (E)-2-decenal and $o$-tolualdehyde (2-methylbenzaldehyde) identified in both beer samples could be the result of autoxidation of unsaturated fatty acids. 


\section{Conclusions}

The fermentation of sweet wort by a brewer's yeast and a Williopsis saturnus yeast was compared. W. saturnus var. mrakii NCYC 500 was able to produce higher concentrations of acetate esters and retain better the original terpenoid content. Fermentation with this yeast could be a novel biotechnological way of increasing the desirable fruity and floral flavour of beer while retaining the characteristic hop aroma of the wort. Given that its fermentative ability of sugars is low, this could be a way of brewing extra-fruity low-alcohol beer.

\section{References}

1. Verstrepen KJ, Derdelinckx G, Dufour JP, Winderickx J, Thevelein JM, Pretorius IS, Delvaux FR. Flavor-active esters: adding fruitiness to beer. J Biosci Bioeng. 2003;96:110-8. http://dx.doi.org/10.1016/S1389-1723(03)90112-5

2. Verstrepen $\mathrm{KJ}$, Van Laere SDM, Vanderhaegen BMP, Derdelinckx G, Dufour JP, Pretorius IS, et al. Expression levels of the yeast alcohol acetyltransferase genes ATF1, LgATF1, and ATF2 control the formation of a broad range of volatile esters. Appl Environ Microbiol. 2003;69:5228-37. http://dx.doi.org/10.1128/AEM.69.9.5228-5237.2003

3. Mason AB, Dufour JP. Alcohol acetyltransferases and the significance of ester synthesis in yeast. Yeast. 2000;16:1287-98. http://dx.doi.org/10.1002/1097-0061(200010)16:14<1287::AIDYEA613>3.0.CO;2-I

4. Saerens SMG, Verstrepen KJ, Van Laere SDM, Voet ARD, Van Dijck P, Delvaux FR, Thevelein JM. The Saccharomyces cerevisiae EHT1 and EEB1 genes encode novel enzymes with medium-chain fatty acid ethyl ester synthesis and hydrolysis capacity. J Biol Chem. 2006;281:4446-56. http://dx.doi.org/10.1074/jbc.M512028200

5. Saerens SMG, Delvaux F, Verstrepen KJ, van Dijck P, Thevelein JM, Delvaux FR. Parameters affecting ethyl ester production by Saccharomyces cerevisiae during fermentation. Appl Environ Microbiol. 2008;74:454-61. http://dx.doi.org/10.1128/AEM.01616-07

6. Wyman C. Handbook on bioethanol: production and utilization. Boca Raton, FL, USA: CRC Press; 1996.

7. Oliver G, Colicchio T. The Oxford companion to beer. Oxford, UK: Oxford University Press; 2011.

8. Vanderhaegen B, Neven H, Coghe S, Verstrepen KJ, Derdelinckx G, Verachtert H. Bioflavoring and beer refermentation. Appl Microbiol Biotechnol. 2003;62:140-50. http://dx.doi.org/10.1007/s00253-003-1340-5

9. James SA, Roberts IN, Collins MD. Phylogenetic heterogeneity of the genus Williopsis as revealed by $18 \mathrm{~S}$ rRNA gene sequences. Int J Syst Evol Microbiol. 1998;48:591-6. http://dx.doi.org/10.1099/00207713-48-2-591

10. Liu SQ, Tsao M. Inhibition of spoilage yeasts in cheese by killer yeast Williopsis saturnus var. saturnus. Int J Food Microbiol. 2009;131:280-2.

http://dx.doi.org/10.1016/j.ijfoodmicro.2009.03.009

11. Yilmaztekin M, Erten H, Cabaroglu T. Production of isoamyl acetate from sugar beet molasses by Williopsis saturnus var. saturnus. J Inst Brew. 2008;114:34-8. http://dx.doi.org/10.1002/j.2050-0416.2008.tb00303.x

12. Inoue $Y$, Fukuda K, Wakai $Y$, Sudsai T, Kimura A. Ester formation by a yeast Hansenula mrakii IFO 0895: contribution of esterase for iso-amyl acetate production in sake brewing. LWT - Food Sci Technol. 1994;27:189-93.

http://dx.doi.org/10.1006/fstl.1994.1037
13. Inoue $Y$, Trevanichi S, Fukuda K, Izawa S, Wakai Y, Kimura A. Roles of esterase and alcohol acetyltransferase on production of isoamyl acetate in Hansenula mrakii. J Agric Food Chem. 1997;45:644-9. http://dx.doi.org/10.1021/jf960648o

14. Yeo HQ, Liu SQ. An overview of selected specialty beers developments, challenges and prospects. Int J Food Sci Technol. 2014;49:1607-18. http://dx.doi.org/10.1111/ijfs.12488

15. NIST08 Mass Spectral Library. Gaithersburg, MD, USA: National Institute of Standards and Technology. Available from: http://chemsw.farhawk.com/NIST08/.

16. Wiley 275 Library. Ringoes, NJ, USA: Scientific Instrument Services, Inc. (SIS). Available from: http://www.sisweb.com/ software/ms/wiley.htm/.

17. Lee PR, Ong YL, Yu B, Curran P, Liu SQ. Evolution of volatile compounds in papaya wine fermented with three Williopsis saturnus yeasts. Int J Food Sci Technol. 2010;45:2032-41. http://dx.doi.org/10.1111/j.1365-2621.2010.02369.x

18. Li X, Yu B, Curran P, Liu SQ. Impact of two Williopsis yeast strains on the volatile composition of mango wine. Int J Food Sci Technol. 2012;47:808-15. http://dx.doi.org/10.1111/j.1365-2621.2011.02912.x

19. Wickerham LJ, Burton KA. Phylogeny and biochemistry of the genus Hansenula. Bacteriol Rev. 1962;26:382-97.

20. De Deken RH. The Crabtree effect: a regulatory system in yeast. Microbiology. 1966;44:149-56. http://dx.doi.org/10.1099/00221287-44-2-149

21. Lagunas R. Sugar transport in Saccharomyces cerevisiae. FEMS Microbiol Rev. 1993;104:229-42. http://dx.doi.org/10.1111/j.1574-6968.1993.tb05869.x

22. Gancedo JM. Yeast carbon catabolite repression. Microbiol Mol Biol Rev. 1998;62:334-61.

23. National Collection of Yeast Cultures (NCYC). Norwich, UK: Institute of Food Research, Norwich Research Park. Available from: http://www.ncyc.co.uk/.

24. Swiegers JH, Saerens SMG, Pretorius IS. The development of yeast strains as tools for adjusting the flavor of fermented beverages to market specifications. In: Havkin-Frenkel D, Belanger FC, editors. Biotechnology in flavor production. Ames, IA, USA: Blackwell Publishing Ltd; 2009. pp.1-55. http://dx.doi.org/10.1002/9781444302493.ch1

25. Montenegro P, Valente IM, Goncalves LM, Rodrigues JA, Barros AA. Single determination of $\alpha$-ketoglutaric acid and pyruvic acid in beer by HPLC with UV detection. Anal Methods. 2011;3:1207-12. http://dx.doi.org/10.1039/C0AY00669F

26. Moreno-Arribas VM, Polo CM. Wine chemistry and biochemistry. New York, NY, USA: Springer; 2009. http://dx.doi.org/10.1007/978-0-387-74118-5

27. Ragazzo-Sanchez JA, Chalier P, Chevalier-Lucia D, Calderon-Santoyo M, Ghommidh C. Off-flavours detection in alcoholic beverages by electronic nose coupled to GC. Sens Actuat B: Chem. 2009;140:29-34. http://dx.doi.org/10.1016/j.snb.2009.02.061

28. Kim WJ, Smit CJB, Rao VNM. Demethylation of pectin using acid and ammonia. J Food Sci. 1978;43:74-8. http://dx.doi.org/10.1111/j.1365-2621.1978.tb09739.x

29. Erten H, Campbell I. The production of low-alcohol wines by aerobic yeasts. J Inst Brew. 2001;107:207-15. http://dx.doi.org/10.1002/j.2050-0416.2001.tb00092.x

30. Hazelwood LA, Daran JM, van Maris AJA, Pronk JT, Dickinson JR. The Ehrlich pathway for fusel alcohol production: a century of research on Saccharomyces cerevisiae metabolism. Appl Environ Microbiol. 2008;74:2259-66. http://dx.doi.org/10.1128/AEM.02625-07 
31. Tehlivets O, Scheuringer K, Kohlwein SD. Fatty acid synthesis and elongation in yeast. Biochim Biophys Acta - Mol Cell Biol Lip. 2007;1771:255-70.

http://dx.doi.org/10.1016/j.bbalip.2006.07.004

32. Malcorps PF, Dufour JP. Short-chain and medium-chain aliphatic-ester synthesis in Saccharomyces cerevisiae. Eur J Biochem. 2005;210:1015-22.

http://dx.doi.org/10.1111/j. 1432-1033.1992.tb17507.x

33. Steinhaus M, Wilhelm W, Schieberle P. Comparison of the most odour-active volatiles in different hop varieties by application of a comparative aroma extract dilution analysis. Eur Food Res Technol. 2007;226:45-55.

http://dx.doi.org/10.1007/s00217-0060-0507-6

34. Nance MR, Setzer WN. Volatile components of aroma hops (Humulus lupulus L.) commonly used in beer brewing. J Brew Distil. 2011;2:16-22.

35. Lermusieau G, Bulens M, Collin S. Use of GC-olfactometry to identify the hop aromatic compounds in beer. J Agric Food Chem. 2001;49:3867-74. http://dx.doi.org/10.1021/jf0101509

36. Kishimoto T, Wanikawa A, Kono K, Shibata K. Comparison of the odor-active compounds in unhopped beer and beers hopped with different hop varieties. J Agric Food Chem. 2006;54:8855-61. http://dx.doi.org/10.1021/jf061342c

37. King AJ, Dickinson JR. Biotransformation of hop aroma terpenoids by ale and lager yeasts. FEMS Yeast Res. 2003;3:53-62. http://dx.doi.org/10.1111/j.1567-1364.2003.tb00138.x

38. Kollmannsberger H, Biendl M, Nitz S. Occurrence of glycosidically bound flavour compounds in hops, hop products and beer. Brew Sci. 2006;59:83-9

39. Takoi K, Koie K, Itoga Y, Katayama Y, Shimase M, Nakayama Y, Watari J. Biotransformation of hop-derived monoterpene alcohols by lager yeast and their contribution to the flavor of hopped beer. J Agric Food Chem. 2010;58:5050-8. http://dx.doi.org/10.1021/jf1000524

40. Morata A, Gómez-Cordovés MC, Colomo B, Suárez JA. Pyruvic acid and acetaldehyde production by different strains of Saccharomyces cerevisiae: relationship with vitisin A and B formation in red wines. J Agric Food Chem. 2003;51:7402-9. http://dx.doi.org/10.1021/jf0304167

41. Nierop Groot MN, de Bont JAM. Conversion of phenylalanine to benzaldehyde initiated by an aminotransferase in Lactobacillus plantarum. Appl Environ Microbiol. 1998;64: 3009-13. 